\title{
MUCK: A toolkit for extracting and visualizing semantic dimensions of large text collections
}

\author{
Rebecca Weiss \\ Stanford University \\ Stanford, CA, 94305 \\ rjweissestanford.edu
}

\begin{abstract}
Users with large text collections are often faced with one of two problems; either they wish to retrieve a semanticallyrelevant subset of data from the collection for further scrutiny (needle-in-a-haystack) or they wish to glean a high-level understanding of how a subset compares to the parent corpus in the context of aforementioned semantic dimensions (forestfor-the-trees). In this paper, I describe $\mathrm{MUCK}^{1}$, an open-source toolkit that addresses both of these problems through a distributed text processing engine with an interactive visualization interface.
\end{abstract}

\section{Introduction}

As gathering large text collections grows increasingly feasible for non-technical users, individuals such as journalists, marketing/communications analysts, and social scientists are accumulating vast quantities of documents in order to address key strategy or research questions. But these groups often lack the technical skills to work with large text collections, in that the conventional approaches they employ (content analysis and individual document scrutiny) are not suitable for the scale of the data they have gathered. Thus, users require tools with the capability to filter out irrelevant documents while drilling-down to the documents that they are most interested in investigating with closer scrutiny. Furthermore, they require the capability to then evaluate their subset in context, as the contrast in attributes between their subset and the full corpora can often address many relevant questions.

This paper introduces a work-in-progress: the development of a toolkit that aids non-technical

\footnotetext{
${ }^{1}$ Mechanical Understanding of Contextual Knowledge
}

users of large text collections by combining semantic search and semantic visualization methods. The purpose of this toolkit is two-fold: first, to ease the technical burden of working with largescale text collections by leveraging semantic information for the purposes of filtering a large collection of text down to the select sample documents that matter most to the user; second, to allow the user to visually explore semantic attributes of their subset in comparison to the rest of the text collection.

Thus, this toolkit comprises two components:

1. a distributed text processing engine that decreases the cost of annotating massive quantities of text data for natural language information

2. an interactive visualization interface that enables exploration of the collection along semantic dimensions, which then affords subsequent document selection and subset-tocorpora comparison

The text processing engine is extensible, enabling the future development of plug-ins to allow for tasks beyond the included natural language processing tasks, such that future users can embed any sentence- or document-level task to their processing pipeline. The visualization interface is built upon search engine technologies to decrease search result latency to user requests, enabling a high level of interactivity.

\section{Related work}

The common theme of existing semantic search and semantic visualization methods is to enable the user to gain greater, meaningful insight into the structure of their document collections through the use of transparent, trustworthy methods (Chuang et al., 2012; Ramage et al., 2009). The desired insight can change depending on the intended task. 
For some applications, users are understood to have a need to find a smaller, relevant subset of articles (or even a single article) in a vast collection of documents, which we can refer to as a needle-in-a-haystack problem. For others, users simply require the ability to gain a broad but descriptive summary of a semantic concept that describes these text data, which we can refer to as a forest-for-the-trees problem.

For example, marketers and social scientists often study news data, as the news constitute a vitally important source of information that guide the agendas of marketing strategy and inform many theories underlying social behavior. However, their interests are answered at the level of sentences or documents that contain the concepts or entities that they care about. This need is often not met through simple text querying, which can return too many or too few relevant documents and sentences. This is an example of a needle-in-ahaystack problem, which has been previously addressed through the application of semantic search (Guha et al., 2003). Much of the literature on semantic search, in which semantic information such as named entity, semantic web data, or simple document categories are added to the individuallevel results of a simple query in order to bolster the relevance of resulting query hits. This type of information has proven to be useful in filtering out irrelevant content for a wide array of information retrieval tasks (Blanco et al., 2011; Pound et al., 2010; Hearst, 1999b; Hearst, 1999a; Liu et al., 2009; Odijk et al., 2012).

Remaining in the same narrative, once a subset of relevant documents has been created, these users may wish to see how the semantic characteristics of their subset contrast to the parent collection from which it was drawn. A marketer may have a desire to see how the tone of coverage in news related to their client's brand compares to the news coverage of other brands of a similar type. A social scientist may be interested to see if one news organization covers more politicians than other news organizations. This is an example of a forest-for-the-trees problem. This type of problem has been addressed through the application of semantic visualization, which can be useful for trend analysis and anomaly detection in text corpora (Fisher et al., 2008; Chase et al., 1998; Hearst and Karadi, 1997; Hearst, 1995; Ando et al., 2000).
The toolkit outlined in this paper leverages both of these techniques in order to facilitate the user's ability to gain meaningful insight into various semantic attributes of their text collection while also retrieving semantically relevant documents.

\section{Overview of System From User Perspective}

The ordering of a user's experience with this toolkit is as follows:

1. Users begin with a collection of unstructured text documents, which must be made available to the system (e.g., on a local or network drive or as a list of URLs for remote content)

2. Users specify the types of semantic detail relevant to their analysis (named entities, sentiment, etc.), and documents are then parsed, annotated, and indexed.

3. Users interact with the visualization in order to create the subset of documents or sentences they are interested in according to semantic dimensions of relevance

4. Once a view has been adequately configured using the visual feedback, users are able to retrieve the documents or sentences referenced in the visualization from the document store

Items 2 and 3 are further elaborated in the sections on the backend and frontend.

\section{Backend}

The distributed processing engine is driven by a task planner, which is a framework for chaining per-document tasks. As diagrammed in figure 1, the system creates and distributes text processing tasks needed to satisfy the user's level of semantic interest according to the dependencies between the various integrated third-party text processing libraries. Additionally, this system does not possess dependencies on additional third-party large-scale processing frameworks or message queueing systems, which makes this toolkit useful for relatively large (i.e. millions of documents) collections as it does not require configuration of other technologies beyond maintaining a document store ${ }^{2}$ and a search index.

\footnotetext{
${ }^{2}$ http://www.mongodb.com
} 


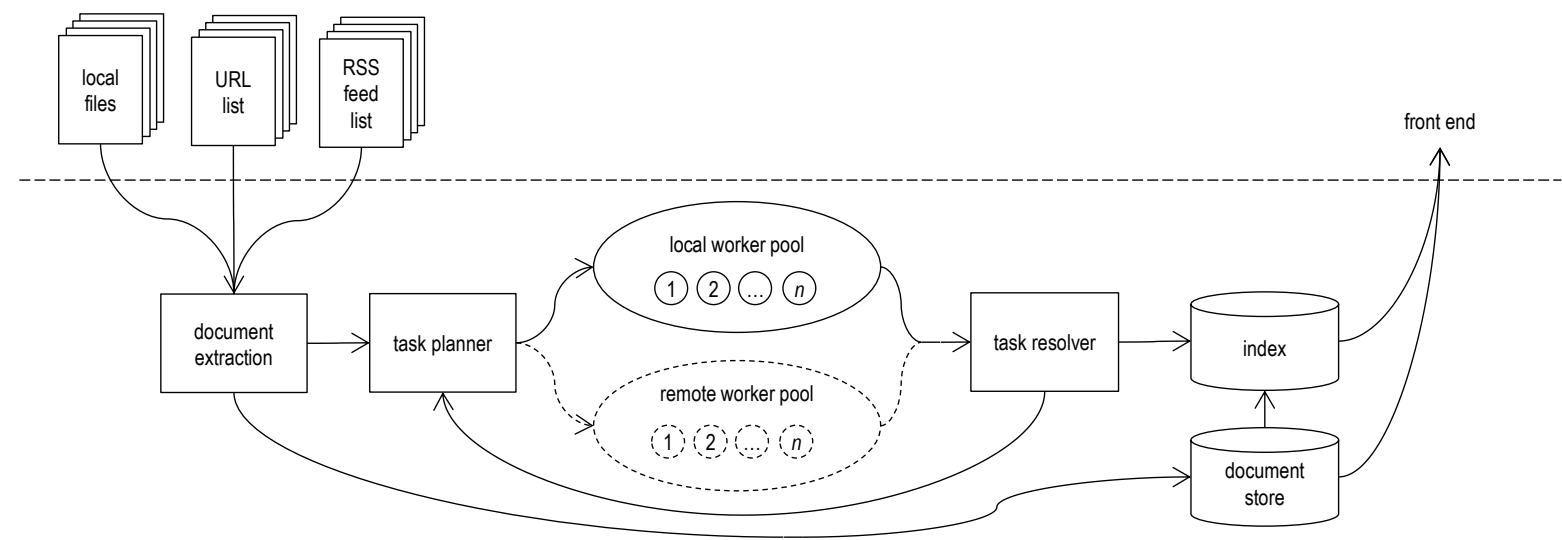

Figure 1: The architecture of the backend system.

Task planner and resolver system The semantic information extraction process occurs via defining a series of tasks for each document. This instantiates a virtual per-document queues of processing tasks. These queues are maintained by a task planner and resolver, which handles all of the distribution of processing tasks through the use of local or cloud resources ${ }^{3}$. This processing model enables non-technical users to describe a computationally-intensive, per-document processing pipeline without having to perform any technical configuration beyond specifying the level of processing detail output desired.

NLP task Currently, this system only incorporates the full Stanford CoreNLP pipeline ${ }^{4}$, which processes each document into its (likely) constituent sentences and tokens and annotates each sentence and token for named entities, parts-of-speech, dependency relations, and sentiment (Toutanova et al., 2003; Finkel et al., 2005; De Marneffe et al., 2006; Raghunathan et al., 2010; Lee et al., 2011; Lee et al., 2013; Recasens et al., 2013; Socher et al., 2013). This extraction process is extensible, meaning that future tasks can be defined and included in the processing queue in the order determined by the dependencies of the new processing technology. Additional tasks at the sentence- or document-level, such as simple text classification using the Stanford Classifier (Manning and Klein, 2003), are included in the development roadmap.

\footnotetext{
${ }^{3}$ http://aws.amazon.com

${ }^{4}$ Using most recent version as of writing (v3.1)
}

\section{Frontend}

A semantic dimension of interest is mapped to a dimension of the screen as a context pane, as diagrammed in figure 2. Corpora-level summaries for each dimension are provided within each context pane for each semantic category, whereas the subset that the user interactively builds is visualized in the focus pane of the screen. By brushing each of semantic dimensions, the user can drilldown to relevant data while also maintaining an understanding of the semantic contrast between their subset and the parent corpus.

This visualization design constitutes a multipleview system (Wang Baldonado et al., 2000), where a single conceptual entity can be viewed from several perspectives. In this case, the semantic concepts extracted from the data can be portrayed in several ways. This system maps semantic dimensions to visualization components using the following interaction techniques:

Navigational slaving Users must first make an initial selection for data by querying for a specific item of interest; a general text query (ideal for phrase matching), a named entity, or even an entity that served in a specific dependency relation (such as the dependent of an nsubj relation). This selection propagates through the remaining components of the interface, such that the remaining semantic dimensions are manipulated in the context of the original query.

Focus + Context Users can increase their understanding of the subset by zooming into a relevant 


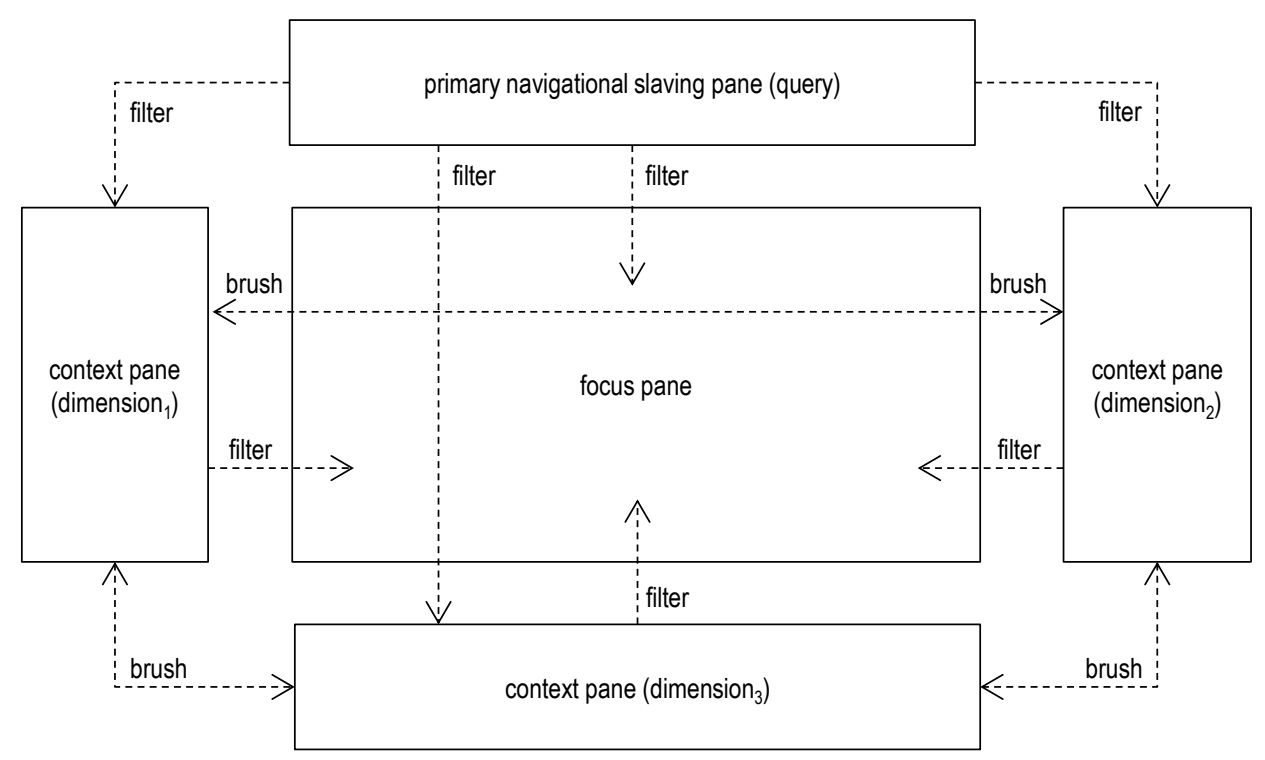

Figure 2: The wireframe of the frontend system.

selection in a semantic dimension (e.g. time).

Brushing Users can further restrict their subset by highlighting categories or ranges of interest in semantic dimensions (e.g. document sources, types of named entities). Brushing technique is determined by whether the semantic concept is categorical or continuous.

Filtering The brushing and context panes serve as filters, which restrict the visualized subset to only documents containing the intersection of all brushed characteristics.

This visualization design is enabled through the use of a distributed search engine ${ }^{5}$, which enables the previously defined interactivity through three behaviors:

Filters Search engines enable the restriction of query results according to whether a query matches the parameters of a filter, such as whether a field contains text of a specific pattern.

Facets Search engines also can return subsets of documents structured along a dimension of interest, such as by document source types (if such information was originally included in the index).

Aggregations Aggregations allow for bucketing of relevant data and metrics to be calculated per

\footnotetext{
${ }^{5}$ http://www.elasticsearch.com
}

bucket. This allows the swift retrieval of documents in a variety of structures, providing the hierarchical representation required for visualizing a subset along multiple semantic dimensions defined above.

Nesting All of these capabilities can be stacked upon each other, allowing for the multiple view system described above.

The visualization components are highly interactive, since the application is built upon a twoway binding design paradigm 6 between the DOM and the RESTful API of the index (Bostock et al., 2011).

\section{Discussion and future work}

This paper presents a work-in-progress on the development of a system that enables the extraction and visualization of large text collections along semantic dimensions. This system is open-source and extensible, so that additional per-document processing tasks for future semantic extraction procedures can be easily distributed. Additionally, this system does not possess requirements beyond maintaining a document store and a search index.

\footnotetext{
${ }^{6}$ http://www.angularjs.org
} 


\section{References}

Rie Kubota Ando, Branimir K Boguraev, Roy J Byrd, and Mary S Neff. 2000. Multi-document summarization by visualizing topical content. In Proceedings of the 2000 NAACL-ANLP Workshop on Automatic Summarization, pages 79-98. Association for Computational Linguistics.

Roi Blanco, Harry Halpin, Daniel M Herzig, Peter Mika, Jeffrey Pound, Henry S Thompson, and T Tran Duc. 2011. Entity search evaluation over structured web data. In Proceedings of the 1st international workshop on entity-oriented search workshop (SIGIR 2011), ACM, New York.

Michael Bostock, Vadim Ogievetsky, and Jeffrey Heer. 2011. $\mathrm{D}^{3}$ data-driven documents. Visualization and Computer Graphics, IEEE Transactions on, 17(12):2301-2309.

Penny Chase, Ray D’Amore, Nahum Gershon, Rod Holland, Rob Hyland, Inderjeet Mani, Mark Maybury, Andy Merlino, and Jim Rayson. 1998. Semantic visualization. In ACL-COLING Workshop on Content Visualization and Intermedia Representation.

Jason Chuang, Daniel Ramage, Christopher Manning, and Jeffrey Heer. 2012. Interpretation and trust: Designing model-driven visualizations for text analysis. In Proceedings of the SIGCHI Conference on Human Factors in Computing Systems, pages 443452. ACM.

Marie-Catherine De Marneffe, Bill MacCartney, Christopher D Manning, et al. 2006. Generating typed dependency parses from phrase structure parses. In Proceedings of LREC, volume 6, pages 449-454.

Jenny Rose Finkel, Trond Grenager, and Christopher Manning. 2005. Incorporating non-local information into information extraction systems by gibbs sampling. In Proceedings of the 43rd Annual Meeting on Association for Computational Linguistics, pages 363-370. Association for Computational Linguistics.

Danyel Fisher, Aaron Hoff, George Robertson, and Matthew Hurst. 2008. Narratives: A visualization to track narrative events as they develop. In Visual Analytics Science and Technology, 2008. VAST'08. IEEE Symposium on, pages 115-122. IEEE.

Ramanathan Guha, Rob McCool, and Eric Miller. 2003. Semantic search. In Proceedings of the 12th international conference on World Wide Web, pages 700-709. ACM.

Marti A Hearst and Chandu Karadi. 1997. Cat-a-cone: an interactive interface for specifying searches and viewing retrieval results using a large category hierarchy. In ACM SIGIR Forum, volume 31, pages 246-255. ACM.
Marti A Hearst. 1995. Tilebars: visualization of term distribution information in full text information access. In Proceedings of the SIGCHI conference on Human factors in computing systems, pages 59-66. ACM Press/Addison-Wesley Publishing Co.

Marti A Hearst. 1999a. Untangling text data mining. In Proceedings of the 37th annual meeting of the Association for Computational Linguistics on Computational Linguistics, pages 3-10. Association for Computational Linguistics.

Marti A Hearst. 1999b. The use of categories and clusters for organizing retrieval results. In Natural language information retrieval, pages 333-374. Springer.

Heeyoung Lee, Yves Peirsman, Angel Chang, Nathanael Chambers, Mihai Surdeanu, and Dan Jurafsky. 2011. Stanford's multi-pass sieve coreference resolution system at the conll-2011 shared task. In Proceedings of the Fifteenth Conference on Computational Natural Language Learning: Shared Task, pages 28-34. Association for Computational Linguistics.

Heeyoung Lee, Angel Chang, Yves Peirsman, Nathanael Chambers, Mihai Surdeanu, and Dan Jurafsky. 2013. Deterministic coreference resolution based on entity-centric, precision-ranked rules.

Shixia Liu, Michelle X Zhou, Shimei Pan, Weihong Qian, Weijia Cai, and Xiaoxiao Lian. 2009. Interactive, topic-based visual text summarization and analysis. In Proceedings of the 18th ACM conference on Information and knowledge management, pages 543-552. ACM.

Christopher Manning and Dan Klein. 2003. Optimization, maxent models, and conditional estimation without magic. In Proceedings of the 2003 Conference of the North American Chapter of the Association for Computational Linguistics on Human Language Technology: Tutorials-Volume 5, pages 8-8. Association for Computational Linguistics.

Daan Odijk, Ork de Rooij, Maria-Hendrike Peetz, Toine Pieters, Maarten de Rijke, and Stephen Snelders. 2012. Semantic document selection. In Theory and Practice of Digital Libraries, pages 215-221. Springer.

Jeffrey Pound, Peter Mika, and Hugo Zaragoza. 2010. Ad-hoc object retrieval in the web of data. In Proceedings of the 19th international conference on World wide web, pages 771-780. ACM.

Karthik Raghunathan, Heeyoung Lee, Sudarshan Rangarajan, Nathanael Chambers, Mihai Surdeanu, Dan Jurafsky, and Christopher Manning. 2010. A multipass sieve for coreference resolution. In Proceedings of the 2010 Conference on Empirical Methods in Natural Language Processing, pages 492-501. Association for Computational Linguistics. 
Daniel Ramage, Evan Rosen, Jason Chuang, Christopher D Manning, and Daniel A McFarland. 2009. Topic modeling for the social sciences. In NIPS 2009 Workshop on Applications for Topic Models: Text and Beyond, volume 5.

Marta Recasens, Marie-Catherine de Marneffe, and Christopher Potts. 2013. The life and death of discourse entities: Identifying singleton mentions. In Proceedings of the 2013 Conference of the North American Chapter of the Association for Computational Linguistics: Human Language Technologies, pages 627-633.

Richard Socher, Alex Perelygin, Jean Y Wu, Jason Chuang, Christopher D Manning, Andrew Y Ng, and Christopher Potts. 2013. Recursive deep models for semantic compositionality over a sentiment treebank. In Proceedings of the Conference on Empirical Methods in Natural Language Processing (EMNLP), pages 1631-1642.

Kristina Toutanova, Dan Klein, Christopher D Manning, and Yoram Singer. 2003. Feature-rich part-ofspeech tagging with a cyclic dependency network. In Proceedings of the 2003 Conference of the North American Chapter of the Association for Computational Linguistics on Human Language TechnologyVolume 1, pages 173-180. Association for Computational Linguistics.

Michelle Q Wang Baldonado, Allison Woodruff, and Allan Kuchinsky. 2000. Guidelines for using multiple views in information visualization. In Proceedings of the working conference on Advanced visual interfaces, pages 110-119. ACM. 